\title{
Hybrid optical coating design for omnidirectional antireflection purposes
}

\author{
Vesna Janicki $^{1,2}$, Steffen Wilbrandt ${ }^{3}$, Olaf Stenzel ${ }^{1}$, Dieter Gäbler ${ }^{1}$, Norbert Kaiser ${ }^{1}$, \\ Alexander Tikhonravov ${ }^{4}$, Mikhail Trubetskov ${ }^{4}$ and Tatiana Amotchkina ${ }^{4}$ \\ ${ }^{1}$ Fraunhofer Institut für Angewandte Optik und Feinmechanik, Optical Coatings Department, \\ Albert-Einstein-Straße 7, 07745 Jena, Germany \\ ${ }^{2}$ Ruđer Bošković Institute, Division of Laser and Atomic Research and Development, \\ Bijenička cesta 54, 10000 Zagreb, Croatia \\ ${ }^{3}$ Friedrich-Schiller-Universität Jena, Institut für Angewandte Physik, Albert-Einstein-Straße \\ 15, 07745 Jena, Germany \\ ${ }^{4}$ Moscow State University, Research Computing Centre, Leninskie Gory, 119992,Moscow, \\ Russia
}

\begin{abstract}
We present a new design for an omnidirectional antireflection coating for the visible spectral range. In contrast to classical designs, it combines homogeneous layers and linear gradient index layers into one hybrid design with a full thickness of approximately $500 \mathrm{~nm}$. The coating may be practically produced based on silicon dioxide as low index material and niobium pentoxide as high index material, while intermediate indices may be obtained from corresponding mixtures.
\end{abstract}

Keywords: antireflective coating, hybrid coating, rugate, gradient index, omnidirectional

\section{Introduction}

Antireflection coatings belong to the most important coating designs. The general idea is to design a coating which diminishes the reflection losses at the surface of an optical component $[1,2]$. For standard situations, practical solutions are well established. Thus, when the antireflection effect is required only at one single wavelength, one may work with single-layer reflection coatings (SLAR), or alternatively utilize V-coatings [1]. The situation becomes more complicated when broadband antireflection is required. A typical solution is the W- 
coating [1]. Recently, a new broadband antireflection coating has been developed which arises from the needle synthesis method, namely the AR-hard ${ }^{\circledR}$ design [3]. As long as normal light incidence is required, the maximum principle [4] states that the most feasible coating design will comprise only two dielectric coating materials with a difference in refractive indices which is as high as possible.

The picture changes completely when oblique incidence is required, or especially in the case of omnidirectional specifications. Then, the application of more than two refractive indices may be of use. In this case, the application of gradient index layers or various kinds of rugate filters may be preferable. It is well known that a negative refractive index gradient diminishes the reflectance of an optical surface, and this way nearly perfect broadband omnidirectional antireflective coatings can be designed [5, 7]. However, if the reflection at the interface substrate/air is to be minimized, the refractive index of the layer should decrease down to the value of air, which makes the coating very demanding for fabrication. Classical rugate filters, on the other hand, show a sinusoidal refractive index profile and are prospective for notch filter designs [6].

Our approach to the omnidirectional antireflection coating is essentially a synthesis of these different approaches. We have designed a coating which consists of a sequence of linear refractive index gradients and homogeneous layers. Figure 1 presents a refractive index profile of general type of a hybrid coating.

Table 1. Sensitivity to errors in individual corner points defined in figure 2(a).

\begin{tabular}{lllll}
\hline & \multicolumn{5}{c}{ Relative change in FOM (\%) } \\
\cline { 2 - 5 } $\begin{array}{l}\text { Corner } \\
\text { point }\end{array}$ & $\begin{array}{l}\text { Change in refractive } \\
\text { index }+1 \%\end{array}$ & $\begin{array}{l}\text { Change in refractive } \\
\text { index }-1 \%\end{array}$ & $\begin{array}{l}\text { Change in } \\
\text { thickness }+1 \%\end{array}$ & $\begin{array}{l}\text { Change in } \\
\text { thickness }-1 \%\end{array}$ \\
\hline A & $\mathbf{2 . 7}$ & 2.4 & - & - \\
B & 0.3 & 0.5 & 0.5 & 0.5 \\
C & $\mathbf{4 . 3}$ & 1.0 & 0.8 & 0.3 \\
D & $\mathbf{7 . 4}$ & 1.7 & 0.8 & 0.5 \\
E & 3.6 & $\mathbf{6 . 7}$ & 2.0 & 0.3 \\
F & - & - & $\mathbf{2 . 0}$ & 0.3 \\
G & - & - & 0.1 & 0.1 \\
H & - & - & 0.1 & 0.1 \\
I & - & - & 2.7 & $\mathbf{7 . 0}$ \\
\hline
\end{tabular}




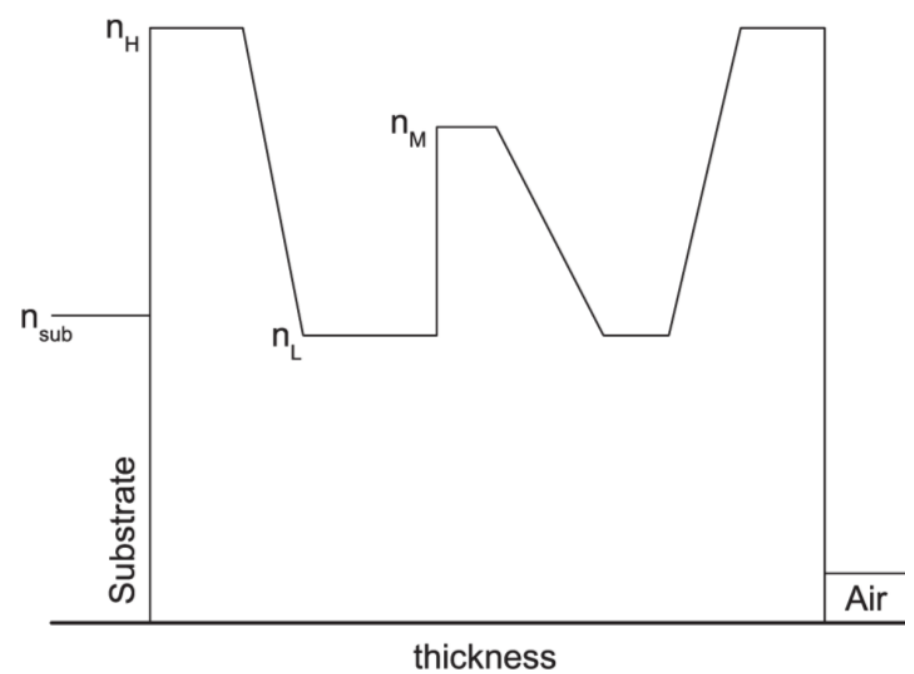

Figure 1. Refractive index profile of a hybrid coating. Here, $n_{\mathrm{H}}, n_{\mathrm{L}}$ and $n_{\mathrm{M}}$ stand, respectively, for the maximal, minimal and some intermediate refractive index in the refractive index profile. The refractive index of the substrate is denoted by $n_{\text {sub. }}$.

\section{Specification and design}

We designed an optical antireflection coating according to the following specifications: wavelength range $480-680 \mathrm{~nm}$; angular range $0^{\circ}-50^{\circ}$; substrate $\mathrm{BK} 7$ glass.

The requirement was to obtain a reflectance $R$ in the mentioned spectral region as low as possible. Here, $R$ is defined as

$$
R \equiv(R \mathrm{~s}+R \mathrm{p}) / 2
$$

$R \mathrm{~s}$ and $R \mathrm{p}$ being the reflectances at $\mathrm{s}$ and $\mathrm{p}$ polarization, respectively. Additionally, it was required that the difference between $R \mathrm{~s}$ and $R \mathrm{p}$ be as small as possible for an angle of incidence of $50^{\circ}$. The calculation has been performed by means of a numerical optimization technique and using the synthesis algorithm recently proposed by Tikhonravov et al [8, 9]. In this software the refractive index profile is approximated by a polyline, i.e. an arbitrary number of corner points $\left(n_{i}, z_{i}\right)$ connected by straight lines representing segments of linear change of refractive index. Here, $n_{i}$ is a refractive index of the layer at corresponding thickness $z_{i}$. For example, the system in figure 1 consists of ten such corner points. In the optimization process the segments are divided into a number of subsegments represented then by homogeneous sublayers all having equal thicknesses and refractive indices equal to the values in the centre of the individual subsegment. The number of sublayers should be sufficient for a reasonably good approximation of a gradient layer. Such defined refractive indices and thicknesses $\left(n_{i}, z_{i}\right)$ are varied until a profile showing the performance that best satisfies the desired specifications is obtained. 
As coating materials, we supposed $\mathrm{Nb}_{2} \mathrm{O}_{5}$ as a high index material, $\mathrm{SiO}_{2}$ as a low index one, and arbitrary mixtures of the two to generate intermediate refractive indices. This approach is based on experimental data on the optical properties of $\mathrm{SiO}_{2} / \mathrm{Nb}_{2} \mathrm{O}_{5}$ mixtures [10]. The refractive index dispersion is automatically considered in the design algorithm.

The result of the design calculation is depicted in figure 2. In figure 2(a) we see the designed refractive index profile at a wavelength of $570 \mathrm{~nm}$. In figure 2(b), the corresponding reflectance is shown for different angles of incidence, together with the corresponding reflectance data for the uncoated substrate. The hybrid coating predicts a very good reflection suppression and well satisfies the specifications defined for $0 \circ$ and $50^{\circ}$. However, a look at figure 2(c), where the average reflectance in the specified spectral region as a function of angle of incidence is presented, shows that even for higher angles of incidence the reflectance of the coated substrate is expected to be lower than that of the uncoated BK7 substrate. The coating thus shows an omnidirectional broadband antireflection effect.

\section{Discussion}

For practical application of the proposed coating, its sensitivity to fabrication errors is of utmost importance. The model of the hybrid coating, where each gradient index segment is divided into ten sublayers of constant refractive indices, was imported into Optilayer software, and the implemented error simulation procedure was performed. A variation of $1 \%$ was permitted in both thicknesses and refractive indices. Only for the two last homogeneous layers of the pure materials no error in refractive index was allowed. The corridor probability has been set to a value of $68.27 \%$. The results of the calculation are presented in figure 3 .

Also, in order to detect which are the most critical corner points, i.e. those most sensitive to errors in fabrication, the coordinates $\left(n_{i}, z_{i}\right)$ of each corner point have been varied individually by $\pm 1 \%$, and the resulting discrepancy from specifications as expressed in terms of the function of merit (FOM) has been checked. In table 1 the corresponding relative changes in the FOM are presented. Thus, the worst performance of the coating was obtained in the case of increased refractive index at point $\mathrm{D}$, decreased thickness of the last layer (point I) or decreased refractive index at point $\mathrm{E}$. 

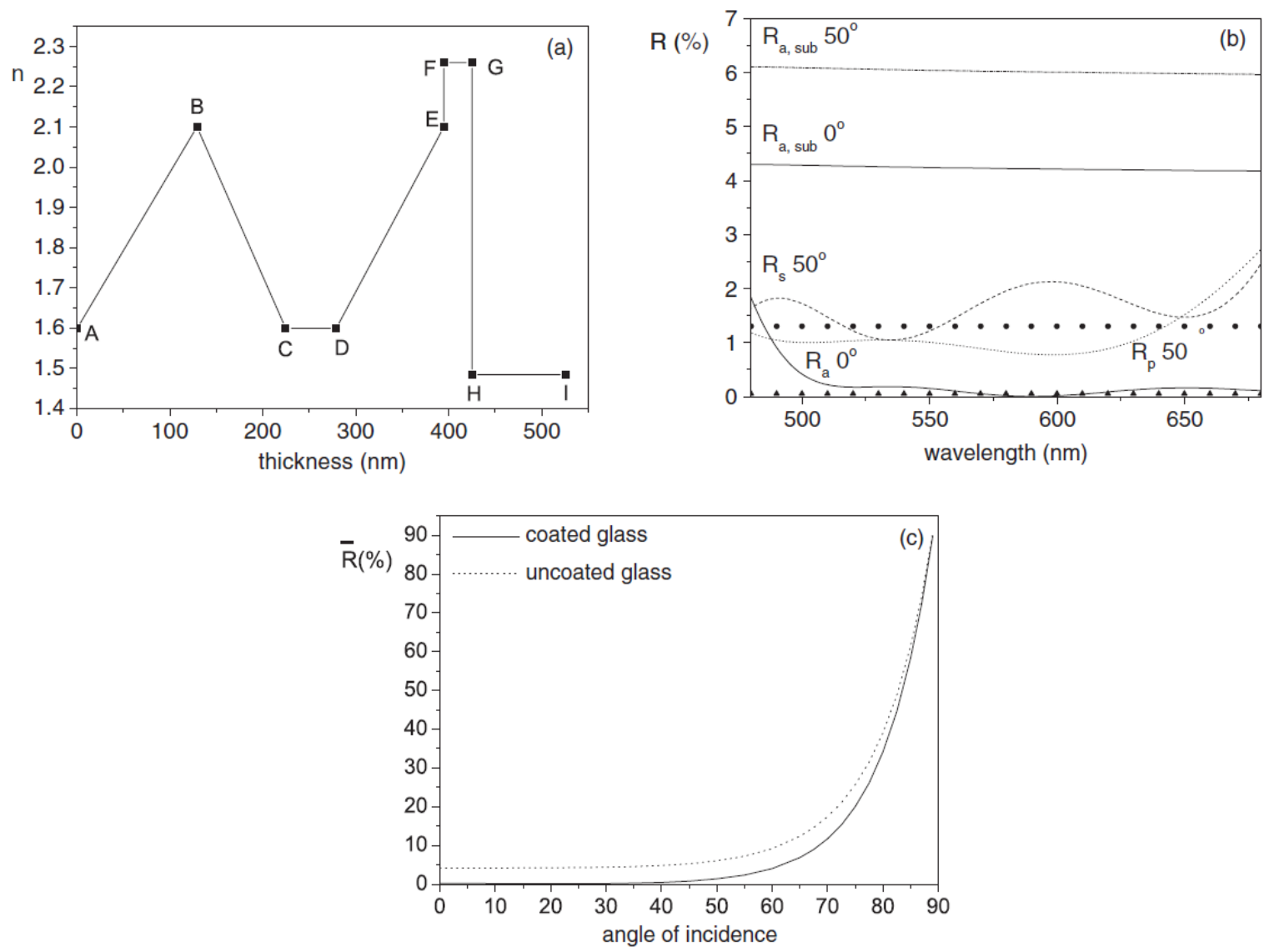

Figure 2. Omnidirectional antireflective coating: (a) refractive index profile at $570 \mathrm{~nm}$, capital letters stand for corner points; (b) optical performance of the design for semi-infinite substrate (no back-side reflection). Triangles represent the reflectance target for average reflectance at $0 \circ$ and circles the reflectance target for $\mathrm{s}$ and $\mathrm{p}$ polarization at the incidence angle of $50^{\circ}$. Rsub stands for isationreflectance of uncoated BK7 glass for unpolarized light. (c) Reflectance average in the spectral range of interest as a functionm of angle of incidence.

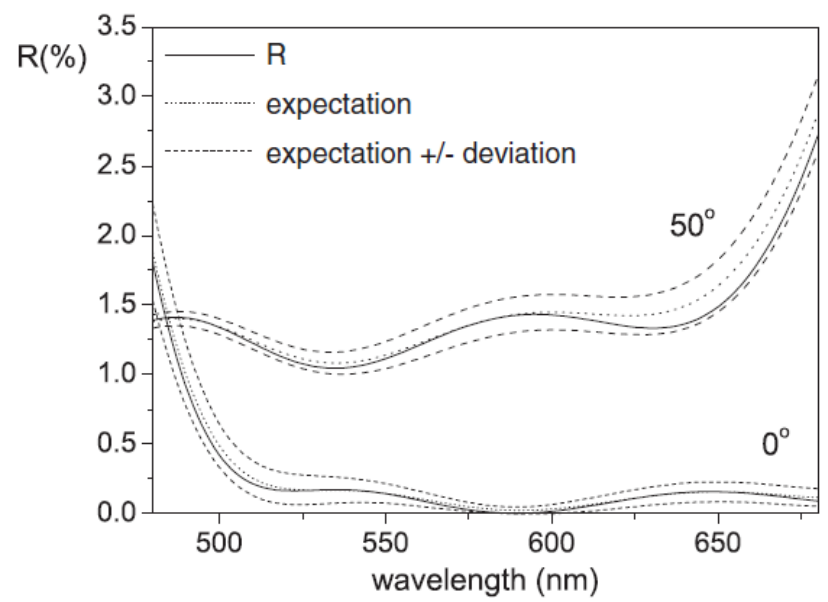

Figure 3. Reflectance variations caused by $1 \%$ random errors of thicknesses and refractive indices. 
The most interesting feature of the proposed design is its small physical thickness of only $526 \mathrm{~nm}$, making it feasible for practical deposition experiments. Indeed, the successful deposition of linear refractive index ramps by co-evaporation of two materials has already been reported [11], and the $\mathrm{R}$ homogeneous layers in the design are standard elements of an optical coating and may be manufactured by any deposition system. It is a particular feature of the proposed design, that the refractive indices are confined to the interval between the high and low index components, and no refractive indices close to that of the ambient (air) are required. Therefore, such hybrid systems are practically affordable thin film systems and highly interesting candidates for omnidirectional antireflection coatings as well as other omnidirectional optical specifications.

\section{Summary and outlook}

We have presented a hybrid optical coating design that is essentially a synthesis from dielectric homogeneous and gradient index layers. It has been demonstrated that such designs show good omnidirectional optical performance. These designs may be practically produced by co-evaporation of a high and a low index coating material. First deposition experiments are in progress in our laboratory and will be published separately.

\section{Acknowledgments}

The authors acknowledge financial support by BMWA, Germany, in terms of the 'rugate' grant. Vesna Janicki wishes to thank the Fraunhofer Society in Germany for a Fraunhofer Fellowship at IOF in Jena.

\section{References}

[1] Thelen A 1989 Design of Optical Interference Coatings (New York: McGraw-Hill)

[2] Macleod H A 1986 Thin-Film Optical Filters (Bristol: Hilger)

[3] Schulz U, Schallenberg U B and Kaiser N 2003 Symmetrical periods for antireflection coatings of plastic optics Appl. Opt. 42 1346-51

[4] TikhonravovA V 1993 Some theoretical aspects of thin-film optics and their applications Appl. Opt. 32 5417-26

[5] SouthwellW H 1983 Gradient-index antireflection coatings Opt. Lett. 8 584-6

[6] Dobrowolski J A, Poitras D, Ma P, Vakil H and Acree M 2002 Toward perfect antireflection coatings: numerical investigation Appl. Opt. 41 3075-83 
[7] Bovard B G 1993 Rugate filter theory: an overview Appl. Opt. 32 5427-42 Rahmlow T D Jr and Lazo-Wasem J E 1997 Rugate and discrete hybrid filter designs SPIE 3133 25-35

[8] TikhonravovA V, TrubetskovM K, Amotchkina T V, KokarevM A, Kaiser N, Stenzel O, Wilbrandt S and Gaebler D 2005 New optimisation algorithm for the synthesis of rugate optical coatings Appl. Opt. submitted

[9] TikhonravovA V, TrubetskovM K, Amotchkina T V, KokarevM A, Kaiser N, Stenzel O and Wilbrandt S 2004 General approach to the synthesis of rugate coatings 6th Int. Conf. 'Prikladnaja Optika' (Sankt Petersburg, Russia, Oct. 2004) vol 3, pp 12-6

[10] Stenzel O,Wilbrandt S, G*abler D, Kaiser N, TikhonravovA V, TrubetskovM K, Amotchkina T V and KokarevM A 2004 Refractive indices of $\mathrm{SiO} 2$ and Nb2O5 mixture films 6th Int. Conf. 'Prikladnaja Optika' (Sankt Petersburg, Russia, Oct. 2004) vol 3, pp 34-8

[11] Kaiser N, Wilbrandt S and Stenzel O 2004 In situ broadband monitoring of optical coatings Proc. 5th Int. Conf. on Coatings on Glass (Saarbr ucken,Germany, July 2004) pp $429-33$ 